\title{
ARTIGOS
}

\section{DISTRIBUIÇÃO GEOGRÁFICA DE PLANORBÍDEOS EM SANTA CATARINA, BRASIL}

\section{Bruno R. Schlemper Junior, Joaquim A. Ferreira Neto, Paulo de Tarso São Thiago, Cinthya Bressan e Alessandro R. do Amarante}

\begin{abstract}
Foi feita uma revisão dos levantamentos malacológicos realizados pela Fundação Nacional de Saúde em 52 municipios de 8 microrregiöes do estado de Santa Catarina, Brasil, de 1981 a 1994. Cinqüenta deles foram positivos para Biomphalaria tenagophila, o único vetor do Schistosoma mansoni identificado nos 94.535 exemplares examinados. Das 1.358 localidades trabalbadas, $617(45,4 \%)$ foram positivas, variando de 4,3\% a $89,4 \%$, por municipio. Os caramujos foram coletados em $0,2 \%$ a $26,3 \%$ das coleções bidricas pesquisadas, por municipio, sendo que 7,1\% (2.013/28.120) serviam de criadouros para o planorbideo. Nos municipios de Araquari, Massaranduba, Joinnille, Jaraguá do Sul e Sãa Francisco do Sul, na microrregião de Joinville, foram encontrados caramujos infectados com $\mathrm{S}$. mansoni. Os dois ültimos são os focos de esquistossomose mansoni mais meridionais do Brasil. Outras pesquisas identificaram a B. peregrina, $\mathrm{B}$. oligoza, B. schrammi, B. straminea e B. occidentalis.
\end{abstract}

Palavras-chaves: Planorbideo. Biomphalaria sp. Esquistossomose mansoni. Santa Catarina. Distribuição geográfica.

Os primeiros registros sobre a ocorrência de planorbídeos em Santa Catarina datam de 1966, com o encontro de Biomphalaria peregrina ${ }^{13}$, e de 1974, com a identificação de $B$. oligoza ${ }^{15}$, em vários municípios. Em 1975 , foi relatada a presença de $B$. tenagophila no Estado ${ }^{16}$ e referida, posteriormente, nos municípios de Blumenau, Itajaí e Joinville ${ }^{17}$. Esta mesma espécie foi registrada em São Francisco do Sul ${ }^{29}$ e Jaraguá do Sul ${ }^{20}$, envolvida nos dois focos de esquistossomose. Em Florianópolis foram encontradas a $B$. tenagophila e a $B$. oligoz $t^{\prime}$. A $B$. straminea foi coletada na capital catarinense e no município de Governador Celso Ramos .

A partir da descoberta, em 1981, do primeiro foco de transmissão da infecção em território catarinense ${ }^{2}$, a Fundação Nacional de Saúde de Santa Catarina-FNS/SC (ex Superintendência de Campanhas de Saúde Pública - SUCAM) iniciou levantamentos malacológicos no estado para determinar a

\footnotetext{
Secretaria Estadual da Saúcle, Fundação Nacional de Saúde e Departamento de Microbiologia e Parasitologia da Universidade Federal de Santa Catarina/UFSC. Florianópolis, SC.

Endereço para correspondência: Prof. Bruno R. Schlemper Junior. Depto. de Microbiologia e Parasitologia/UFSC. 88.040-900 Florianópolis, SC.

Recebido para publicação em 11/02/95.
}

espécie, as áreas de ocorrência dos transmissores e a infecção pelo $S$. mansoni. Uma vez que estes dados encontram-se apenas em relatórios internos da FNS, o presente trabalho objetiva divulgar os seus resultados e contribuir para um melhor conhecimento da distribuição geográfica dos vetores da esquistossomose mansoni no estado, pois a infecção apresenta algum potencial de expansão para o extremo sul do Brasil ${ }^{19}$. Foi também revisada a literatura sobre planorbídeos em Santa Catarina, bem como obtidas informações sobre coletas feitas por diferentes pesquisadores.

\section{MATERIAL E MÉTODOS}

O levantamento malacológico foi realizado pela FNS/SC no período de 1981 a 1994 em todas as localidades dos municípios selecionados de Santa Catarina. Previamente era feito o cadastramento da população, levantamento das condições sanitárias e mapeamento de todas as coleções hídricas que pudessem se constituir em possiveis criadouros para os caramujos. Utilizando-se concha apropriada fazia-se a coleta nas coleções hídricas, com intervalos de, aproximadamente, 50 passadas (cerca de $25 \mathrm{~m}$ ), entre cada estação.

Os caramujos eram contados e examinados na própria localidade, um a um, com identificação da espécie pela observação macroscópica da concha e submetidos, em 
Schlemper Junior BR, Ferreira Neto JA, Thiago PTS, Bressan C, Amarante AR. Distribuição geográfica de planorbideos em Santa Catarina, Brasil. Revista da Sociedade Brasileira de Medicina Tropical 29:411-418, set-out, 1996

seguida, à compressão entre placas de vidro, em grupos de 3 a 5 exemplares, para pesquisa de cercárias de $S$. mansoni em microscópio estereoscópico. Periodicamente, lotes de caramujos eram enviados para o Laboratório de Esquistossomose do Departamento de Microbiologia e Parasitologia da Universidade Federal de Santa Catarina e, eventualmente, para o Instituto de Biociências da Universidade de São Paulo para confirmação da espécie e identificação das cercárias

\section{RESULTADOS}

A Tabela 1 mostra o resultado do levantamento malacológico realizado em 52 municípios de 8 microrregiões de Santa Catarina. Apenas em São Bento do Sul e Itapoá não foram encontrados caramujos transmissores do S. mansoni. Todos os demais 50 municípios foram positivos para $B$. tenagopbila, a única espécie identificada nos 94.535 exemplares examinados (Figura 1). Em 12 destes municípios, os percentuais de localidades com presença do transmissor variaram de $4,3 \%$ a $20,0 \%$, em 11 de $22,2 \%$ a $40,0 \%$, em 12 de $43,1 \%$ a $60,0 \%$ e 15 apresentaram indices acima de 63,7\%, chegando a valores máximos de $88,6 \%$ e $89,4 \%$. Em termos gerais, em $45,4 \%$ das localidades examinadas $(617 / 1.358)$ foram coletados exemplares de $B$. tenagophila, os quais estavam presentes em $7,1 \%$ $(2.013 / 28.120)$ das coleçôes hídricas pesquisadas. Em 21 municípios os percentuais de criadouros existentes variaram de $0,1 \%$ a $5,0 \%$, em 18 de $5,1 \%$ a $10,0 \%$ e em 11 de $10,1 \%$ a $26,3 \%$.

Das 7 microrregiões com caramujos, a de Tijucas, com $14,5 \%$ e a de Tabuleiro, com $27,8 \%$, tiveram as menores taxas de localidades positivas (Tabela 1). Nestas duas, 1,1\% e 2,3\% das coleções hídricas, respectivamente, tinham a presença do vetor. Nas demais 5 microrregiôes com $B$. tenagopbila os indices gerais de localidades positivas variaram de $43,1 \%$ a $54,3 \%$ e de coleções hídricas -com criadouros de $6,7 \%$ a $8,8 \%$.

Cinco municípios, localizados na microrregião de Joinville, no litoral norte de Santa Catarina, apresentaram $B$. tenagophila eliminando cercárias de S. mansoni: Araquari, Massaranduba, Jaraguá do Sul, Joinville e São Francisco do Sul.

\section{DISCUSSÃO}

Os insuficientes conhecimentos existentes sobre a real distribuiçâo geográfica dos transmissores da esquistossomose no Brasil devem-se às dificuldades de acesso a determinadas áreas e ao pequeno número de pesquisadores interessados ${ }^{1.4}$. Certamente este último fator foi relevante para que, conforme a Tabela 2, o primeiro registro da presença de planorbídeos em Santa Catarina fosse, apenas, de 1966, ocasião em que foram coletados exemplares de $B$. peregrina em vários municípios de diferentes microrregiões do Estado ${ }^{13}$. Também nos estados vizinhos do Paraná e Rio Grande do Sul esta espécie está amplamente disseminada ${ }^{13}{ }^{21}$. A $B$. oligoza tem sua ocorrência em Santa Catarina referida a partir de $1974^{15}$, quase sempre em baixa densidade. Em duas localidades de Florianópolis foram coletados apenas 22 exemplares $^{6}$ e sua presença já foi também registrada nos estados vizinhos do Paraná e Rio Grande do Sul ${ }^{15}$. Por sua vez, a B. schrammi foi identificada, em Santa Catarina, em 1978, numa única localidade no município de Itajaí (WL Paraense: comunicação pessoal, 1994), parecendo constituir-se na primeira e única referência no sul do Brasil, tendo em vista que, até o momento, ela não foi registrada nos outros dois Estados ${ }^{16}$.

Interessante foi o primeiro encontro de $B$. straminea, em 1989, em Santa Catarina ${ }^{8}$ uma vez que, possivelmente, sua introdução tenha se dado por meio do transporte de peixes e plantas ornamentais. O encontro desta espécie em aquários na cidade de Florianópolis e nos tanques de criação e cultivo dos mesmos no municipio catarinense de Governador Celso Ramos $^{8}$, parece corroborar este fato. Situação similar ocorreu em São Paulo, onde a $B$. straminea foi introduzida através do comércio de peixes oriundos de estações de piscicultura do Ceará. Esta espécie foi encontrada em São José do Rio Preto (São Paulo) em tanques de criação de peixes e cultivo de plantas ornamentais, de onde parece ter se dispersado para outros municípios pelo comércio de plantas aquáticas ${ }^{23}$. A $B$. straminea já havia sido detectada no Paraná ${ }^{16}$ e no Rio Grande do Sul ${ }^{31}$ e, com a inclusão de Santa Catarina, completa-se seu encontro em todos os estados brasileiros ${ }^{16}$.

A primeira e única identificação de $B$. occidentalis em Santa Catarina é de 1991, com a coleta de 1.100 exemplares na localidade de 
Schlemper Junior BR, Ferreira Neto JA, Thiago PTS, Bressan C, Amarante AR. Distribuição geográfica de planorbideos em Santa Catarina, Brasil. Revista da Sociedade Brasileira de Medicina Tropical 29:411-418, set-out, 1996.

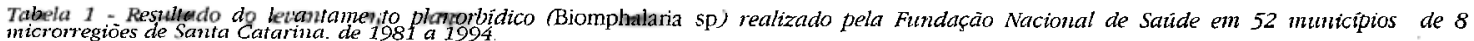
microrregiöes de Santa Catarina. de 1981 a 1994.

\begin{tabular}{|c|c|c|c|c|c|c|}
\hline \multirow{2}{*}{$\begin{array}{l}\text { Microrregião } \\
\text { município }\end{array}$} & \multicolumn{2}{|l|}{ Localidade } & \multirow[b]{2}{*}{ Ano } & \multicolumn{2}{|l|}{ Coleção hídrica } & \multirow{2}{*}{$\begin{array}{l}\text { B. renagopbila } \\
\text { coletada }\end{array}$} \\
\hline & pesquisada/positiva & $\%$ & & pesquisada/positiva & $\%$ & \\
\hline \multicolumn{7}{|l|}{ Micronegiăo de Joinville } \\
\hline São Francisco do Sul* & $31 / 11^{* *}$ & 35.4 & $1981,1982,1983$ & $360 / 77$ & 21,3 & 4. 017 \\
\hline Joinville* & $58 / 34^{* *}$ & 58.6 & $1982,1984,1985,1986,1994$ & $2.128 / 163$ & 7,6 & 21.111 \\
\hline Araquari* & $16 / 12^{* *}$ & 75.0 & $1983.1988,1989$ & $1.025 / 66$ & 6,4 & 3.734 \\
\hline Jaraguá do Sul* & $67 / 29^{* *}$ & 43.2 & 1986,1991 & $1,442 / 115$ & 7,9 & 4.624 \\
\hline Massaranduba* & $30 / 12$ & 40,0 & 1985 & $929 / 75$ & 8,1 & 8.477 \\
\hline Ganuva & $13 / 1$ & 7,7 & 1985 & $417 / 1$ & 0,2 & 12 \\
\hline Itapoá & $10 / 0$ & 0,0 & 1985 & $190 / 0$ & 0.0 & 0 \\
\hline Balneário Barra do Sul & $5 / 1$ & 20,0 & 1983,1988 & $268 / 2$ & 0,7 & 46 \\
\hline Guaramirim & $29 / 21$ & 72.4 & 1985.1994 & $915 / 92$ & 10,0 & 13.220 \\
\hline Schroeder & $23 / 2$ & 8.7 & $1985,1986.1991$ & $327 / 11$ & 3,4 & 1.443 \\
\hline Corupá & $29 / 11$ & 37.9 & 1986 & $472 / 29$ & 6,1 & 1.140 \\
\hline Subtotal & $311 / 134$ & 43,1 & & $8.473 / 631$ & 7,4 & 57.824 \\
\hline \multicolumn{7}{|c|}{ Micromegião de São Bento do Sul } \\
\hline São Bento do Sul & $10 / 0$ & 0,0 & 1986 & $144 / 0$ & 0,0 & 0 \\
\hline Subtotal & $10 / 0$ & 0,0 & & $144 / 0$ & 0,0 & 0 \\
\hline \multicolumn{7}{|l|}{ Micronegiāo de Blumenau } \\
\hline Blumenau & $92 / 42$ & 45,6 & 1993.1994 & $1.760 / 91$ & 5,2 & 3.187 \\
\hline Rodeio & $19 / 17$ & 89,4 & 1994 & $260 / 29$ & 11,1 & 744 \\
\hline Luiz Alves & $27 / 4$ & 14,8 & 1986 & $398 / 4$ & 1,0 & 47 \\
\hline Pomerode & $33 / 19$ & 57,5 & 1986 & $660 / 36$ & 5,4 & 702 \\
\hline Timbó & $17 / 13$ & 76,5 & 1986 & $429 / 38$ & 8,8 & 722 \\
\hline Benedito Novo & $49 / 5$ & 10,2 & 1987 & $539 / 11$ & 2,0 & 164 \\
\hline Doutor Pedrinho & $13 / 2$ & 15,4 & 1987 & $162 / 2$ & 1,2 & 22 \\
\hline Gaspar & $44 / 39$ & 88.6 & 1988 & $1.856 / 217$ & 11,7 & 3.132 \\
\hline Rio dos Cedros & $33 / 5$ & 15.1 & 1987 & $424 / 14$ & 3,3 & 221 \\
\hline Brusque & $39 / 19$ & 48,7 & 1988.1989 & $1.016 / 82$ & 8,1 & 1.279 \\
\hline Apiúna & $32 / 15$ & 46,9 & 1993 & $713 / 27$ & 3,8 & 196 \\
\hline Ascurra & $12 / 9$ & 75.0 & 1993. 1994́ & $184 / 15$ & 8,1 & 404 \\
\hline Indainl & $33 / 23$ & 69,6 & 1994 & $752 / 44$ & 5,8 & 349 \\
\hline Subtotal & $443 / 212$ & 47,8 & & $9.153 / 610$ & 6.7 & 11.169 \\
\hline \multicolumn{7}{|l|}{ Micromegião de Ilajaí } \\
\hline Itajấ & $20 / 15$ & 75.0 & 1987 & $724 / 61$ & 8,4 & 1.845 \\
\hline Barra Velha & $11 / 7$ & 63,7 & 1984. 1989 & $608 / 16$ & 2,6 & 769 \\
\hline São João do Itaperiú & $13 / 4$ & 30,8 & 1984,1989 & $556 / 8$ & 1.4 & 278 \\
\hline Ilhota & $17 / 11$ & 64,7 & 1982. 1987 & $472 / 57$ & 12.2 & 2.356 \\
\hline Navegantes & $11 / 2$ & 18,2 & 1986 & $195 / 2$ & 1,0 & 168 \\
\hline Penha & $7 / 4$ & 57.1 & 1986 & $136 / 9$ & 6,6 & 506 \\
\hline Piçarras & $10 / 1$ & 10.0 & 1986 & $203 / 1$ & 0,4 & 53 \\
\hline Balneário Camboriú & $11 / 8$ & 72,7 & 1987 & $346 / 91$ & 26,3 & 3.393 \\
\hline Camboriú & $25 / 17$ & 68.0 & 1987 & $395 / 74$ & 18.7 & 1.451 \\
\hline Itapema & $15 / 12$ & 80,0 & 1991 & $311 / 41$ & 13,2 & 2.317 \\
\hline Porto Belo & $15 / 5$ & 33.3 & 1990,1991 & $260 / 11$ & 4,2 & 592 \\
\hline Bombinhas & $9 / 3$ & 33.3 & 1991 & $70 / 4$ & 5.7 & 67 \\
\hline Subrotal & $164 / 89$ & 54.3 & & $4.276 / 375$ & 8,8 & 13.805 \\
\hline \multicolumn{7}{|l|}{ Microryegião de Tijucas } \\
\hline Tijucas & $21 / 7$ & 33.3 & 1991 & $380 / 8$ & 2,1 & 92 \\
\hline Canelinha & $25 / 2$ & 8.0 & 1991 & $338 / 2$ & 0.6 & 46 \\
\hline Sāo João Batista & $23 / 1$ & 4.3 & 1991 & $260 / 1$ & 0.3 & 12 \\
\hline Subtotal & $69 / 10$ & 14.5 & & $978 / 11$ & 1,1 & 150 \\
\hline Micromegiào de Florianópo & & & & & & \\
\hline Governador Celso Ramos & $12 / 2$ & 16,6 & 1991 & $261 / 26$ & 10,0 & 546 \\
\hline Biguaçú & $49 / 14$ & 28.6 & 1991 & $663 / 20$ & 3,0 & 622 \\
\hline Săo José & $35 / 21$ & 60,0 & 1991,1992 & $454 / 59$ & 13,0 & 2.264 \\
\hline Santo Amaro da Imperatriz & $27 / 16$ & 59.2 & 1993 & $301 / 32$ & 10,6 & 779 \\
\hline Pálhoça & $48 / 34$ & 70,8 & 1992 & $761 / 85$ & 11.2 & 3.921 \\
\hline Paulo Lopes & $22 / 7$ & 31.8 & 1992,1993 & $272 / 9$ & 3,3 & 495 \\
\hline Subtotal & $193 / 94$ & 48.7 & & $2.712 / 231$ & 8,5 & 8.627 \\
\hline Micromegião do Tabuleiro & & & & & & \\
\hline Águas Momas & $18 / 5$ & 27.8 & 1993 & $221 / 5$ & 2,3 & 165 \\
\hline Subtotal & $18 / 5$ & 27.8 & & $221 / 5$ & 2,3 & 165 \\
\hline Microrregiāo de Tubaräo & & & & & & \\
\hline Garopaba & $18 / 4$ & 22.2 & 1992 & $162 / 7$ & 4,3 & 64 \\
\hline Inbituba & $28 / 15$ & 53.6 & 1992 & $208 / 40$ & 19.2 & 494 \\
\hline Săo Martinbo & $12 / 8$ & 66.7 & 1993 & $412 / 21$ & 5.1 & 251 \\
\hline Imanií & $41 / 24$ & 58.5 & 1993 & $744 / 33$ & 4.4 & 915 \\
\hline Laguna & $51 / 22$ & 43.1 & 1993 & $637 / 49$ & 7,7 & 1.071 \\
\hline Subtotal & $150 / 73$ & 48,7 & & $2.163 / 150$ & 6,9 & 2.795 \\
\hline Total & $1.358 / 617$ & 45,4 & & $28.120 / 2.013$ & 7.1 & 94.535 \\
\hline
\end{tabular}

* Município com exemplares de $B$. tenagophila positivos para Scbistosoma mansoni.

* Dados referentes a coletas realizadas antes da aplicação de moluscicida nas localidades. 
Schlemper Junior BR, Ferreira Neto JA, Thiago PTS, Bressan C, Amarante AR. Distribuição geográfica de planorbideos em Santa Catarina, Brasil. Revista da Sociedade Brasileira de Medicina Tropical 29:411-418, set-out, 1996.

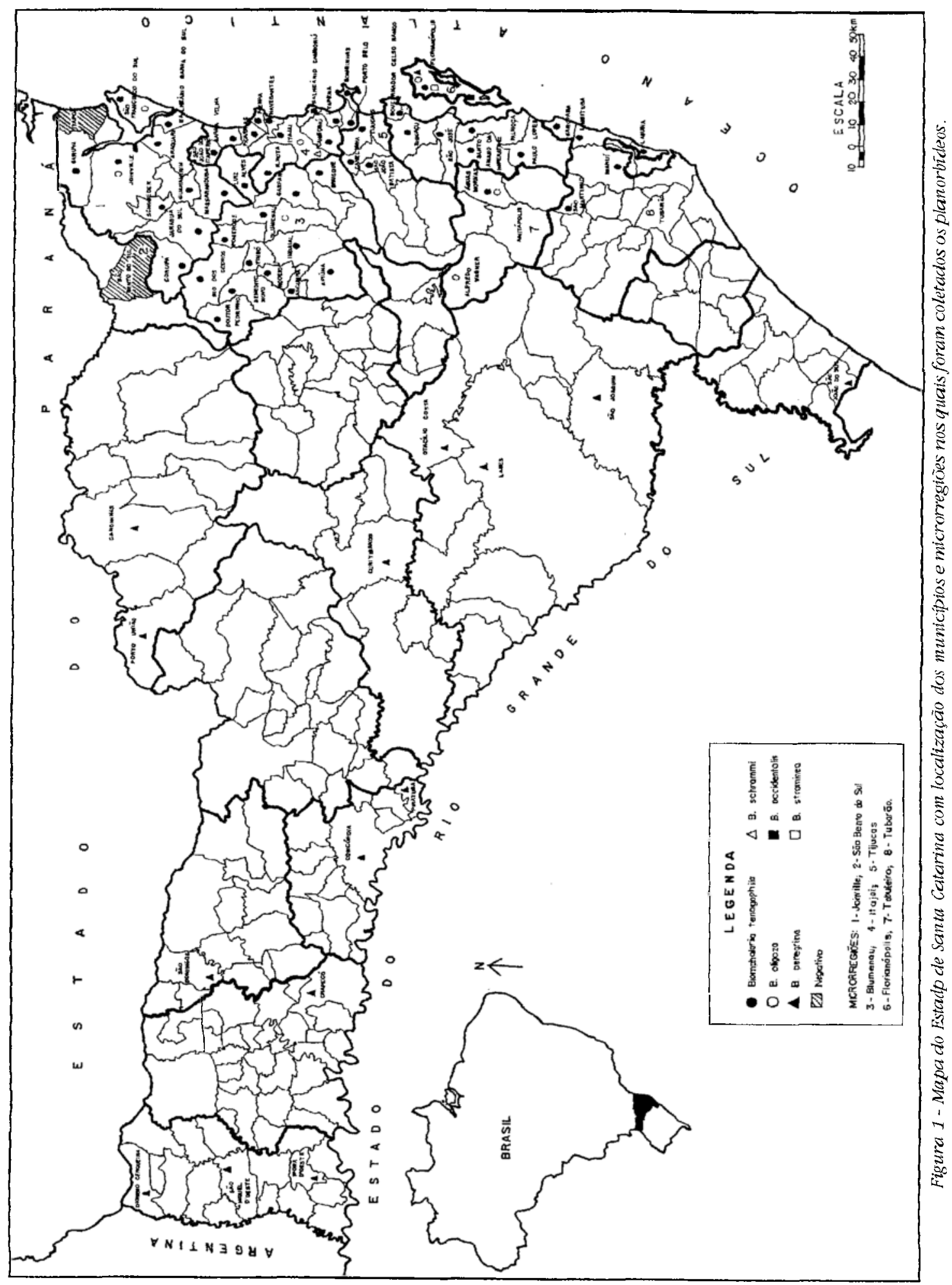


Schlemper Junior BR, Ferreira Neto JA, Thiago PTS, Bressan C, Amarante AR. Distribuição geográfica de planorbideos em Santa Catarina, Brasil. Revista da Sociedade Brasileira de Medicina Tropical 29:411-418, set-out, 1996.

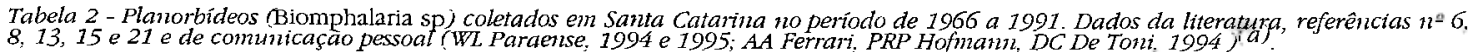

\begin{tabular}{ll}
\hline Planorbídeo & \multicolumn{1}{c}{ Município } \\
\hline B. tenagopbila Francisco & \\
& Itajaí* $^{*}$ Blumenau* $^{*}$ \\
& \\
Joinville* \\
\\
B. oligoza
\end{tabular}

Itajaí*

Blumenau* Acara Ano Planorbídeos coletados

$1978 \quad 316$

Paulas 1978

Dom Bosco 1978

Fortaleza 1977

Itoupava Norte 1977

Velha 1977

Àgua Verde 1977

Badenfürt $\quad 1977$

Iririú 1977

Itaum 1977

Floresta $\quad 1977$

$\mathrm{km} 4$

Nova Brasília $\quad 1977$

Salāo Reis 1977

Afonso Voss $\quad 1977$

Boa Vista $\quad 1977$

Córrego Grande 6

Alto Ribeirão** 1991

Itajuba

?

Canastieiras*

Rio Tavares"

Rio Tavares

Ribeirãoda Itha 6

?15 1974

Dom Bosco 1978

Badenfürt $\quad 1977$

Progresso 1977

Velha 1977

Garcia 1977

Itoupava Central 1977

Joinville

Iririữ

1977

Costa e Silva* 1977

$\mathrm{Km} \mathrm{4^{* }} 1977$

Itaum* 1977

?15 1974

São Francisco.do Sul

Alfredo Wagner (ex Barracão) ${ }^{15}$

Acaraí 1978

? 1974

Queçaba 1974

Dom Bosco 1978

Alto Ribeirão 1991

Caieira do Norte 1989

Anchieta 1991

Alto Ribeirão 1991

Palmeira ?

? 1988

Conceição 1966

\section{Concórdia 21}

São João do Sul ${ }^{13}$

Porto União 13

Florianópolis, Canoinhas, Chapecó, Lages,

Santa Cruz do Timbó 1966

\section{6}

125

?

38

211

?

575

387

337

410

218

522

66

1.303

?

28

?

?

8

14

!

!

119

?

227

17

25

?

16

(1)

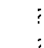

؛

1.100

231

166

59

São Joaquim, Curitibanos, Dionisio Cerqueira,

Piraruba, Iporã do Oeste, São Domingos,

São Miguel do Oeste ${ }^{13}$

(a) Não inclui os resultados do levantamento da FNS.

Planorbideos coletados e identificados, respectivamente, por :

* JA Ferreira Neto; WL Paraense: comunicação pessoal, 1994 .

** AA Ferrati, PRP Hofmann, DC De Toni: comunicaçäo pessoal, 1994; AA Ferrari e WL. Paraense.

*** WL Paraense; WL Paraense: comunicaçāo pessoal, 1995. 
Schlemper Junior BR, Ferreira Neto JA, Thiago PTS, Bressan C, Amarante AR. Distribuição geográfica de planorbideos em Santa Catarina, Brasil. Revista da Sociedade Brasileira de Medicina Tropical 29:411-418, set-out, 1996.

Alto Ribeirão, na Ilha de Santa Catarina, Florianópolis (AA Ferrari, PRP Hofmann, DC De Toni : comunicação pessoal, 1994). Com este registro, o limite sul de ocorrência desta espécie no Brasil passa a ser Santa Catarina, tendo em vista que ela já havia sido assinalada no Paraná ${ }^{17}$.

Quanto a B. tenagopbila, sua presença no estado do Paraná remonta a $1949^{10}$, no Rio Grande do Sul, a pelo menos $38 \operatorname{anos}^{18}$ e, em Santa Catarina, o primeiro encontro data de $1975^{16}$. A partir de 1977 (WL Paraense: comunicação pessoal, 1994) e de 1981, com a pesquisa realizada pela FNS/SC, sua presença passou a ser registrada em 50 municípios. Mais recentemente, a $B$. tenagopbila foi encontrada no município de Florianópolis, no bairro de Córrego Grande ${ }^{7}$ e na localidade de Alto Ribeirão (AA Ferrari, PRP Hofmann, DC De Toni: comunicação pessoal, 1994), elevando para 51 o número de municípios com este planorbídeo no Estado. No Brasil, a $B$. tenagopbila ocorre em uma faixa contínua da região litorânea, desde o município de Caravelas, na Bahia, até o Chuí, no extremo sul do Rio Grande do Sul ${ }^{18}$. A aparente existência de uma falha nesta distribuição ininterrupta, em Santa Catarina, situada ao sul do município de Blumenau, foi atribuída à possível ausência de pesquisas nesta área ${ }^{18}$. De fato, somente a partir de 1987, com o início dos trabalhos da FNS nesta região, é que se demonstrou que esta lacuna não existe, uma vez que todos os municípios pesquisados ao sul de Blumenau foram positivos para B. tenagopbila (Figura 1).

Os resultados do levantamento desenvolvido pela FNS demonstram a grande dispersão da B. tenagophila em Santa Catarina, presente em $96,1 \%$ dos municípios trabalhados, em $45,4 \%$ das localidades pesquisadas e em $7,1 \%$ das coleções hídricas examinadas. Em 5 microrregiões, os percentuais de localidades positivas $(43,1 \%$ a $54,3 \%)$ e de criadouros existentes $(6,9 \%$ a $8,8 \%)$ apresentaram valores altos e diferiram muito pouco de uma para outra área. As elevadas taxas e a homogênea distribuição do caramujo nestas microrregiões possivelmente são decorrentes de serem elas regiōes litorâneas banhadas por 9 bacias hidrográficas (dos rios Cubatão [Nortel, Itapocu, Itajaí-Açu, Tijucas, Biguaçu, Cubatão [Sull, da Madre, d'Una e Tubarão), que ocupam uma área de $28.549 \mathrm{~km}^{2}$ e com um comprimento dos cursos dos rios da ordem de $44.409 \mathrm{~km}$. Estes rios, pertencentes à vertente atlântica, possuem um perfil longitudinal acidentado no curso superior e, no curso inferior - região dos municípios trabalhados pela FNS - geralmente formam meandros com baixas declividades. Estes rios de planície drenam as águas oriundas dos contrafortes ocidentais das serras do Mar, Geral e outras, e percorrem inúmeros vales sujeitos a grandes inundações ${ }^{1}$. Dispersão semelhante parece ter ocorrido em vários municípios da bacia do Tietê, no estado de São Paulo, com a B. tenagopbila e B. peregrina, levadas para pontos distantes não só pelas águas dos rios, mas também pelas inundações ${ }^{22}$.

Os municípios trabalhados situam-se em região de mata atlântica, com temperaturas médias anuais variando de 18 a $22^{\circ} \mathrm{C}$, precipitação pluviométrica anual entre 1.400 a $2.200 \mathrm{~mm}$, distribuida durante todo 0 ano, e umidade relativa anual de 75 a $85 \%$, ou até mesmo superior. Em quase todos os municípios, o clima é do tipo úmido, com exceção da região de Joinville, em que é super úmido e onde se localizam os focos de esquistossomose mansoni no estado. A maior parte da área pesquisada situa-se ao nível do mar, excetuandose alguns poucos municipíos localizados nas serras litorâneas com até $800 \mathrm{~m}$ de altitude ${ }^{1}$.

Além de ter sido verificada a infecção natural de $B$. tenagophila em 5 municípios, no levantamento realizado pela $\mathrm{FNS} / \mathrm{SC}$, estudos experimentais revelaram que esta espécie, oriunda de São Francisco do Sul, facilmente se infecta com cepas de $S$. mansoni do mesmo local (11 infectados entre 12 examinados) ${ }^{2}$ ou com a BH-MG $(56,5 \%-69 / 106)^{7}$. Em outros experimentos, exemplares de B. tenagopbila de São Francisco do Sul foram expostos a 3 cepas de $S$. mansoni, apresentando os seguintes índices de infecção: $2,5 \%$ com a cepa SFC (São Francisco do Sul), 25,7\% com a Beberibe (Pernambuco) e 66,0\% com a BHMG (Belo Horizonte) ${ }^{12}$. Em contrapartida, 102 exemplares de $B$. tenagophila de Florianópolis, do bairro Córrego Grande, não se infectaram experimentalmente com a cepa $\mathrm{BH}-\mathrm{MG}^{7}$ e nem com a cepa Beberibe 3 . Estudos semelhantes com a cepa SJ (São José dos Campos, SP) de $S$. mansoni e B. tenagopbila, do Rio Grande do Sul, mostraram que o helminto infectou $2,08 \%$ dos caramujos (4/188) do município de Tramandaí ( $B$. tenagophila tenagophila) e foi incapaz de causar infecção em 123 exemplares oriundos de Guaiba (B. tenagopbila guaibensis) ${ }^{19}$. 
Schlemper Junior BR, Ferreira Neto JA, Tbiago PTS, Bressan C, Amarante AR. Distribuição geogräfica de planorbideos em Santa Catarina, Brasil. Revista da Sociedade Brasileira de Medicina Tropical 29:411-418, set-out, 1996.

A disseminação da esquistossomose é devido, principalmente, à migração de pessoas infectadas para áreas indenes mas com presença de transmissores potenciais ${ }^{19}$. Esta parece ter sido a situação ocorrida em relação aos focos de São Francisco do Sul, em 1981² e Jaraguá do Sul, em $1991^{20}$, no norte de Santa Catarina. Por sua vez, os relatórios técnicos da FNS de 1985 e 1986 referem a ocorrência de 12 casos autóctones da infecção no bairro Bom Retiro, no município catarinense de Joinville. Neste mesmo local, no entanto, de acordo com os registros da FNS, foram negativos para $S$. mansoni 4.502 exemplares de $B$. tenagophila coletados entre 1985 e 1988 , bem como 720 exames coprológicos de escolares, em 1994. A identificação do primeiro caso autóctone da helmintíase em São Valentim, no Rio Grande do Sul ${ }^{11}$, também merece ser melhor investigada. Estes dados reforçam a preocupação de que a doença possa vir a se dispersar para o extremo sul do país ${ }^{19}$. Por outro lado, a $B$. tenagopbila, por sua ampla disseminação e capacidade de se infectar, natural e experimentalmente, se constitui, até o momento, na única espécie responsável pela transmissão do parasito nos focos existentes e relacionada com a possivel expansão da doença nesta região do Brasil. Sugere-se a continuidade dos levantamentos malacológicos no estado e a adoção de medidas adequadas pelos órgãos competentes para controlar os atuais focos e evitar o aparecimento de novas áreas de transmissão da esquistossomose mansoni em Santa Catarina.

\section{SUMMARY}

$A$ retision of the malacological suneys camied out in Santa Catarina, Brazil, by the National Health Foundation in 52 counties of 8 microregions between 1981 and 1994 was made. Fifty of them were positive for Biomphalaria tenagophila, the only scbistosome vector identified in the 94,535 specimens collected. of 1,358 districts, $617(45.4 \%)$ were positive to the vector, varying from $4.3 \%$ to $89.4 \%$ per municipality. The percentages of water bodies with planorbids varied from $0.2 \%$ to $26.3 \%$ and, of the total 28,120 examined, 2,013 (7.1\%) were positive. Snails infected with cercariae of Schistosoma mansoni were found in the counties of Araquari, Massaranduba, Joini ille, Jaraguá do Sul and São Francisco do Sul, the last two constituting the soutbernmost focus of schistosomiasis mansoni in Brazil. Other planorbid species identified were $\mathrm{B}$. peregrina, B. oligoza, B. schrammi, B. straminea and $\mathrm{B}$. occidentalis
Key-words: Planorbid. Biomphalaria sp. Schistosomiasis. Santa Catarina. Geographical distribution.

\section{AGRADECIMENTOS}

Ao Dr. Almir Vieira Stadler, Coordenador Regional e Sr. Evaristo Floriano de Souza, Chefe de Informações Epidemiológicas, da FNS/SC, pela cessão dos relatórios e boletins técnicos e ao Dr. W. Lobato Paraense pela revisão do manuscrito.

\section{REFERÊNCIAS BIBLIOGRÁFICAS}

1. Atlas Escolar de Santa Catarina. Secretaria de Estado de Coordenação e Planejamento, Subsecretaria de Estudos Geográficos e Estatísticos.Aerofoto Cruzeiro S.A. Rio de Janeiro, 1991.

2. Bernardini OJ, Machado MM. Esquistossomose mansoni em SantaCatarina: isolamento do Scbistosoma mansoni do primeiro foco de transmissão ativa em São Francisco do Sul. Arquivos Catarinenses de Medicina 10:213, 1981.

3. Bernardini OJ, Machado MM, Espíndola KS, Gomes ZRD. Estudo da susceptibilidade de Biomphalaria tenagopbila Orbigny, 1835, procedentes do Córrego Grande, Florianópolis, Santa Catarina, a cepa Beberibe (PE) de Scbistosoma mansoni Sambon, 1907. In: Caderno de Resumos da II Semana da Pesquisa da Universidade Federal de Santa Catarina. Florianópolis, p. 255, 1994.

4. Corrêa RR, Murgel JMT, Piza JT, Ramos AS, Dias LCS, Morais LVC, Rosário FF. Dispersão de Biomphalaria straminea, hospedeira intermediária do Schistosoma mansoni através da distribuição de peixes. Revista de Saúde Pública de São Paulo 4: 117-127, 1970.

5. Cunha Neto AG. Biomphalaria straminea em Pôrto Alegre, Rio Grande do Sul, Brasil. Nota Prévia. Atas da Sociedade de Biologia do Rio de Janeiro 15: 151, 1972.

6. Espíndola KS, Hofmann PRP, Machado MM. Preliminary survey of the planorbidic fauna in Santa Catarina island, Santa Catarina, Brazil. Memórias do Instituto Oswaldo Cruz 85: 375 , 1990.

7. Espíndola KS, Machado MM, Hofmann PRP. Natural and experimental infection of planorbids from the island of Santa Catarina (Brazil). Revista do Instituto de Medicina Tropical de São Paulo 34: 289-294, 1992. 
Scblemper Junior BR, Ferreira Neto JA, Thiago PTS, Bressan C, Amarante AR. Distribuição geográfica de planorbideos em Santa Catarina, Brasil. Revista da Sociedade Brasileira de Medicina Tropical 29:411-418, set-out, 1996.

8. Ferrari AA, Hofmann PRP. First register of Biomphalaria straminea Dunker, 1848 , in Santa Catarina state. Revista do Instituto de Medicina Tropical de São Paulo 34: 33-35, 1992.

9. Ferreira Neto JA, Cavalcanti JR. Esquistossomose autóctone em Santa Catarina, Brasil. In: Anais do XIX Congresso da Sociedade Brasileira de Medicina Tropical. Rio de Janeiro, p. 98-99, 1983.

10. Lobo AGS, Luz E. Contribuição ao conhecimento da distribuição geográfica dos planorbídeos no Estado do Paraná. Revista Brasileira de Malariologia e Doenças Tropicais 6: 546-554, 1954

11. Louzada JLZ. Esquistossomose mansônica (Primeiro caso autóctone no Rio Grande do Sul). Revista Brasileira de Medicina 30: 533-535, 1973.

12. Machado MM. Estudo do comportamento de Biomphalaria tenagophila de São Francisco do Sul (SC) frente a diferentes cepas de Schistosoma mansoni. Int: Caderno de Resumos da II Semana da Pesquisa da Universidade Federal de Santa Catarina. Florianópolis, p. 256, 1994.

13. Paraense WL. The synonymy and distribution of Biomphalaria peregrina in the neotropical region. Revista Brasileira de Biologia 26:269-296, 1966.

14. Paraense WL. Fauna planorbídica do Brasil. In: Lacaz CS, Baruzzi RG, Siqueira Junior W. (eds) Introdução à Geografia Médica do Brasil. Editora Edgar Blücher e Universidade de São Paulo, São Paulo, p. 213-239, 1972.

15. Paraense WL. Biomphalaria oligoza n.n. for Tropicorbis philippianus (Dunker) sensu Lucena. Revista Brasileira de Biologia 34: 379-386, 1974.
16. Paraense WL. Estado atual da sistemática dos planorbídeos brasileiros (Mollusca, Gastropoda). -Arquivos do Museu Nacional do Rio de Janeiro 55: 105-128, 1975.

17. Paraense WL. Biomphalaria occidentalis, sp.n. from South America (Mollusca Basommatophora Pulmonata). Memórias do Instituto Oswaldo Cruz 76: 199-211, 1981.

18. Paraense WL. Modernos conhecimentos sobre Esquistossomose Mansônica. Distribuição dos caramujos no Brasil. Anais da Academia Mineira de Medicina 14 (supl. de 1983 e 1984): 117-128, 1986.

19. Paraense WL, Corrêa LR. Probable extension of schistosomiasis mansoni to southernmost Brazil. Memórias do Instituto Oswaldo Cruz 82: 577 , 1987.

20. São Thiago P. T. Sobre um novo foco de esquistossomose mansoni em Santa Catarina. Revista da Sociedade Brasileira de Medicina Tropical 27 (supl. 1): 192, 1994.

21. Teles HMS, Pereira PAC, Richinitti LMZ. Distribuição de Biomphalaria (Gastropoda, Planorbidae) nos Estados do Rio Grande do Sul e Santa Catarina, Brasil. Revista de Saúde Pública de São Paulo 25: 350-352, 1991.

22. Vaz JF Distribuição e dipersão de Biomphalaria tenagophila (d'Orbigny, 1835) (GastropodaPulmonata). Ciência e Cultura 41: 14-27, 1989.

23. Vaz JF, Elmor MRD, Gonçalves LMC. Levantamento planorbídico do Estado de São Paulo: $8^{a}$ Região Administrativa (Grande Área de São José do Rio Preto). Revista do Instituto de Medicina Tropical de São Paulo 34: 527-534, 1992. 